\title{
El impacto de la Ley de cuotas sobre la representación de las mujeres en la política argelina
}

The impact of the Quota law on women's representation in Algerian politics

\section{Carmen GARRATÓN MATEU}

Universidad de Granada

cgarraton@ugr.es

https://orcid.org/0000-0002-9095-8209

Recibido 07/07/2020. Aceptado 24/11/2021.

Para citar este artículo: Carmen GARRATÓN MATEU (2021): "El impacto de la ley de cuotas sobre la representación de las mujeres en la política argelina" en Revista de Estudios Internacionales Mediterráneos, 30, pp. 62-81

Para acceder a este artículo: https://doi.org/10.15366/reim2021.30.004

\section{Resumen:}

La promulgación de la ley de cuotas de 2012 incrementó notablemente la representación de las mujeres argelinas en las asambleas electas. En el presente trabajo analizamos la evolución de la participación femenina en política antes y después de la adopción de dicha ley. Asimismo, prestamos atención al contexto en el que se gestó el texto legal y realizamos un balance de sus consecuencias más inmediatas. Concluimos con una reflexión acerca de la efectividad de la estrategia adoptada y de sus perspectivas de cara a la promoción de la mujer en las esferas de decisión y en la sociedad en general.

Palabras clave: política argelina/ ley de cuotas Argelia/ mujer y política/ elecciones Argelia/ igualdad de géneros/

\section{Abstract:}

The promulgation of the quota law in 2012 has increased notably Algerian women's representation in elective assemblies. This paper analyses the evolution of female representativeness before and after the adoption of the above-mentioned law. Furthermore, it highlights the context in which this legal text was developed, underlining its most immediate consequences. It concludes with a reflection on the effectiveness of the chosen measure and on its perspectives to promote women at decision-making levels and in society.

Keywords: Algerian politics/ Algerian quota law/ Women and policy/ Algerian elections/ Gender equality/ 


\section{Introducción}

La subrepresentación de las mujeres en la esfera política constituye una asignatura pendiente en la lucha por la igualdad de géneros, pese a que las mujeres representan más de la mitad de la población mundial. Esta realidad obedece con frecuencia a la existencia de leyes, prácticas o estereotipos sexistas discriminatorios, que dificultan o impiden su incorporación a la vida pública, obviando que su presencia es imprescindible para el buen funcionamiento democrático y para el logro de un desarrollo sostenible. En Argelia, la escasa participación de las mujeres en los asuntos políticos ha sido la tónica dominante, especialmente en circunscripciones remotas donde la sociedad es más reacia a introducir cambios. Estos datos contrastan paradójicamente con su exitosa incorporación a la educación universitaria y con su presencia a la cabeza de ciertos ámbitos profesionales como la enseñanza, la salud o la judicatura (Saad-Zoy et al, 2010: 37).

Para hacer frente a esta situación, el gobierno argelino promulgó, el 12 de enero de 2012, la Ley Orgánica ${ }^{1}$ que fija las modalidades para aumentar las posibilidades de acceso de la mujer a la representación en las asambleas electas, conocida comúnmente como Ley de cuotas, con el propósito de dar respuesta a las recomendaciones internacionales y a las reivindicaciones femeninas. Esta ley establece un sistema de cuotas mínimas de candidatas en las listas electorales de los partidos políticos, delegando en estos toda la responsabilidad para el cumplimiento de la norma.

El presente trabajo analiza la participación política en Argelia desde una perspectiva de género (Lamas, 1996), con el objetivo de valorar la idoneidad y los efectos de la adopción del sistema de cuotas como estrategia para revertir la situación de inferioridad de las mujeres. Con esta finalidad, desde el punto de vista metodológico, se realiza un estudio diacrónico de la representatividad alcanzada por las mujeres argelinas en las asambleas electas, tomando como base la valoración de los resultados obtenidos en los sucesivos comicios nacionales y locales. Asimismo, se lleva a cabo un análisis en profundidad del texto de la Ley de cuotas y se propone una reflexión crítica acerca de su efectividad ante el reto de alcanzar la igualdad real de géneros en el ámbito político.

\section{La participación de las mujeres en la política en los primeros años de consolidación de la nación}

Desde la independencia argelina las mujeres han estado facultadas para postularse como candidatas en todas las elecciones, incluidas las presidenciales, sin embargo, su incorporación a la política ha sido lenta y desigual como se desprende de los sucesivos resultados electorales.

Los primeros comicios que se convocaron, el 20 de septiembre de 1962, tenían como objetivo elegir a los miembros de la Asamblea Constituyente. Estas elecciones fueron más bien una "parodia de democracia", ya que los candidatos propuestos se presentaron en una lista única y cerrada. En esta ocasión, todavía con el recuerdo reciente de la guerra de liberación, la presencia femenina se

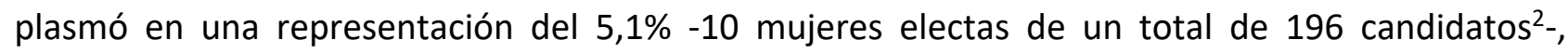
contribuyendo a crear al menos la ilusión de que éstas formaban parte de la escena política. La

\footnotetext{
${ }^{1}$ Qānūn 'uḍ̂ī raqn 03-12, mu'arraj fĩ 18 șafar 'ām 1433 al-muwāfiq 12 yanā'yir sana 2012, yuhadidu kayfiyyāt tawsī' huzūūẓ tamt̄îl al-mar’a fĩ al-maȳâlis al-muntajiba (vers. Francesa: Loi organique n. 12-03 du 18 Safar 1433 correspondant au 12 janvier 2012 fixant les modalités augmentant les chances d'accès de la femme à la représentation dans les assemblées élues, Journal Officiel de la République Algérienne (JORA), n. 01, 14 janvier 2012).

${ }^{2}$ Los resultados de las elecciones legislativas a la APN en este trabajo provienen en lo sucesivo del siguiente enlace: http://www.apn.dz/fr/ [consulta: 26 de octubre de 2020].
} 
Asamblea Constituyente resultante no elaboró en realidad el texto constitucional, que fue encargado a una asamblea paralela externa (Fougères, 1963: 9).

La Constitución de $1963^{3}$, instauró un sistema de partido único en favor del FLN y estableció la Asamblea Nacional como única cámara de representación parlamentaria elegible por sufragio universal directo, libre y secreto cada cinco años (Fougères, 1963: 15). En este texto ya se recogía, al igual que harán las sucesivas Constituciones, el principio de igualdad de todos los ciudadanos de ambos sexos en derechos y deberes instando, en el Preámbulo, a "acelerar la emancipación de la mujer con el fin de asociarla a la gestión de los asuntos públicos y al desarrollo del país". Sin embargo, las propias dirigentes de la Unión Nacional de Mujeres Argelinas (UNFA), dependiente del FLN y canalizadora de las reivindicaciones femeninas, consideraron por entonces que las mujeres aún no estaban preparadas para asumir tareas de índole política (Pérez Beltrán, 1997: 265). De hecho, la creación de la UNFA "supuso la prohibición de cualquier otra opción femenina o feminista que no fuera la puramente oficial", contribuyendo a promover el estatus tradicional de las mujeres como esposas y madres (Pérez Beltrán, 1998: 80). Esto explicaría que, tras la euforia de los primeros momentos, la mayoría de las mujeres se reintegraran a sus hogares, quedando la política femenina en manos de una "elite muy minoritaria y sin eficacia" (Graba, 2013: 7).

En estos primeros años de consolidación de la nación, la cuestión de la mujer ya formaba parte del discurso de los poderes públicos como puso de manifiesto la Carta de Argel ${ }^{4}$. Conforme a este texto, durante siglos se había mantenido a la mujer argelina en un "estado de inferioridad que se tendía a justificar en base a concepciones retrógradas o a interpretaciones erróneas del islam". Por ello, el FLN se atribuía la responsabilidad de suprimir los frenos que impidiesen la evolución de la mujer y de apoyar la acción de las organizaciones femeninas para acabar con esa "mentalidad negativa". El partido consideraba fundamental que la mujer argelina participara efectivamente en la acción política y en la construcción del socialismo militando y asumiendo responsabilidades en el partido y en las organizaciones nacionales, poniendo sus energías al servicio del país ${ }^{5}$.

La prueba, no obstante, de que este discurso oficial no llegó a surtir efecto la constituyen los resultados de los primeros comicios legislativos de 1964, convocados para elegir a los candidatos del FLN a la Asamblea Nacional. En este caso la presencia femenina, lejos de incrementarse, descendió y sólo fueron elegidas 2 mujeres de 138 diputados, resultando una ínfima tasa de representación femenina del 1,4\%. Esta legislatura, prevista inicialmente para cinco años, resultó bastante efímera debido al golpe de Estado de 19 de junio de 1965, protagonizado por Houari Boumédiène. Durante esta nueva etapa la posición del partido en el poder con respecto a las mujeres fue muy ambigua, contribuyendo de facto a limitar su rol en la sociedad.

El discurso pronunciado por Boumédiène, el 8 de marzo de 1966, Día Internacional de la Mujer, dejaba patente esta postura. Tras afirmar que la cuestión de los derechos de la mujer estaba "resuelta por haberlos adquirido participando en la lucha de liberación", matizaba que la evolución de la mujer no significaba "imitación de la mujer occidental" y se posicionaba firmemente diciendo "no" a esta clase de evolución porque la sociedad argelina era una sociedad "islámica y socialista" y esta evolución no debía ser la causa de la "corrupción" de dicha sociedad (Pérez Beltrán, 1997: 251252). La nueva Constitución, que verá la luz en $1976^{6}$ recalcaba que la mujer debía "participar plenamente en la edificación socialista y en el desarrollo nacional" (art. 81), aunque no se proponía ninguna medida concreta por lo que en las siguientes elecciones a la Asamblea Popular Nacional (APN), celebradas el 25 de febrero de 1977, pese a un ligero repunte, no se observaron cambios y

\footnotetext{
${ }^{3}$ http://www.conseil-constitutionnel.dz/index.php/fr/1963 [consulta: 26 de octubre de 2020].

${ }^{4}$ Conjunto de documentos procedentes del primer congreso del FLN celebrado entre los días 16 y 21 de abril de 1964.

${ }^{5}$ Carta de Argel, segunda parte, capítulo III, artículo 8 "Le rôle de la femme algérienne".

${ }^{6}$ http://www.conseil-constitutionnel.dz/index.php/fr/1976 [consulta: 26 de octubre de 2020].
} 
sólo resultaron electas 9 mujeres de 273 diputados, lo que elevó la representatividad femenina únicamente al 3,3\%.

El anuncio del gobierno, en 1979, de la creación de una comisión para redactar un anteproyecto de código de familia, será el detonante para que empiecen a surgir los primeros movimientos organizados de mujeres opuestos a la política oficial aunque la existencia de la UNFA constituye un obstáculo para sacar adelante cualquier otra iniciativa que no estuviera en consonancia con el ideario del partido único suponiendo además un pasaje obligado para cualquier candidata a un puesto electo (Benghabrit-Remaoun, 2012: 135). Por ello, ni las movilizaciones femeninas, ni la amenaza inminente del código vendrán acompañadas por un incremento del número de mujeres en las instancias representativas como quedó patente en el período legislativo comprendido entre 1982-1987, en el que la representatividad femenina sólo alcanzó el 2,10\%, con solo 6 mujeres electas de 285 diputados de la Asamblea Popular Nacional.

El Código de la familia de 1984 salió finalmente adelante a pesar de que desde el primer momento fue cuestionado por juristas y asociaciones de mujeres de diferentes tendencias, que lo consideraron una ley discriminatoria y anticonstitucional (Remaoun, 1999: 130) conforme a la cual "La mujer puede ser presidente de la República, pero debe contar con la asistencia jurídica de su padre para contraer matrimonio" (Graba, 2013: 6). La consecuencia inmediata de la promulgación del código será "el nacimiento de los movimientos feministas realmente estructurados" (Pérez Beltrán, 1997: 314). Sin embargo, este activismo no se traducirá en una emergencia política de mujeres con vocación de cambiar las leyes como volvió a poner en evidencia el resultado de las elecciones de 1987, con sólo 7 mujeres electas y una tasa de participación femenina del 2,3\%, en un total de 296 diputados.

Todo este período, estuvo marcado por la preeminencia exclusiva del FLN como partido único lo que limitaba las posibilidades de presentar alternativas fuera del marco oficial. La representatividad de las mujeres en la política en general resultó fluctuante y prácticamente testimonial, tal y como se puede apreciar en el siguiente gráfico, que recoge los resultados de las elecciones a la Asamblea Constituyente y los sucesivos comicios a la Asamblea Popular Nacional:

[Fig. 1] Resultados por géneros de las elecciones nacionales celebradas entre 1962 y 1992.

\begin{tabular}{|c|c|c|c|c|c|c|}
\hline \multicolumn{2}{|c|}{$\begin{array}{c}\text { Asamblea Constituyente } \\
1962\end{array}$} & $\begin{array}{l}\text { LEGISLATURA } \\
\text { (APN) }\end{array}$ & 1964-1965 & 1977-1982 & $1982-1987$ & 1987-1992 \\
\hline Hombres & 186 & Diputados & 136 & 264 & 279 & 289 \\
\hline Mujeres & 10 & Diputadas & 2 & 9 & 6 & 7 \\
\hline Miembros totales & 196 & Total escaños & 138 & 273 & 285 & 296 \\
\hline \% Mujeres & $5,1 \%$ & $\%$ Mujeres & $1,4 \%$ & $3,3 \%$ & $2,1 \%$ & $2,3 \%$ \\
\hline
\end{tabular}


Por otra parte, a nivel local, desde la independencia, también se aprecia una masculinización casi exclusiva de las candidaturas electorales. En las primeras elecciones a las Asambleas Populares Comunales $(\mathrm{APC})^{7}$, y por la influencia de la participación femenina en la Guerra de Liberación Nacional, existía una ligera tendencia más favorable a la participación política de las mujeres, por lo que, en 1967, el porcentaje de candidatas electas fue del 2,03\%, descendiendo sin embargo al 0,44\% en las elecciones de 1971. En 1975, coincidiendo con la declaración del Año Internacional de la mujer, se produce un pequeño repunte y resultan elegidas un porcentaje de $1,38 \%$ candidatas, para volver a caer en picado de nuevo en 1979 , donde solo consiguen una representatividad del $0,8 \%$ :

[Fig. 2] Resultados por géneros de las elecciones comunales entre 1967 y 1979.

\begin{tabular}{|l|c|c|c|c|}
\hline ELECCIONES APC & 1967 & 1971 & 1975 & 1979 \\
\hline Diputadas & 208 & 46 & 160 & 97 \\
\hline Diputados & 10.031 & 10.375 & 11.360 & 11.978 \\
\hline Total diputados & 10.239 & 10.424 & 11.520 & 12.075 \\
\hline \% Mujeres & $2,03 \%$ & $0,44 \%$ & $1,38 \%$ & $0,80 \%$ \\
\hline
\end{tabular}

Fuente.: Elaboración propia con los datos procedentes de Pérez Beltrán (1997:260).

En cuanto a la representación femenina alcanzada en la Asambleas Populares de Wilaya (APW), la situación es bastante similar. En 1969 resultan electas un 3,78\% de candidatas, y a partir de este dato, el porcentaje subirá ligeramente al 4,06\% en 1974, para volver a decaer hasta el 3\% en 1979:

[Fig. 3] Resultados por géneros de las elecciones de wilayas entre 1967 y 1979.

\begin{tabular}{|l|c|c|c|}
\hline ELECCIONES APW & 1969 & 1974 & 1979 \\
\hline Diputadas & 25 & 45 & 37 \\
\hline Diputados & 636 & 1.063 & 1.196 \\
\hline Total diputados & 661 & 1.108 & 1.233 \\
\hline \% Mujeres & $3,78 \%$ & $4,06 \%$ & $3,00 \%$ \\
\hline \multicolumn{3}{|c|}{ Fuente: Ibidem } \\
\end{tabular}

En la década de los 80, sólo 50 mujeres fueron elegidas a las Asambleas Populares de Wilaya (APW) y 60 a las Asambleas Populares Comunales (APC) (Saad-Zoy et al, 2010: 38), muy lejos de alcanzar la paridad entre sexos.

Entre los factores que influyen en esta escasa feminización de la política destacan, por una parte, la falta de interés de las organizaciones de masa como la UNFA en concienciar a las mujeres y, por otra parte, la mentalidad del electorado de la época donde la mayoría de los hombres se mostraba hostil a la presencia de candidatas en las diferentes asambleas y las propias mujeres afirmaban no sentirse interesadas por las cuestiones políticas (Pérez Beltrán, 1997: 266-267).

\footnotetext{
${ }^{7}$ Las APC y las APW constituyen las circunscripciones en las que se desarrollan los comicios locales. La wilaya es una división administrativa similar al departamento francés. Cada wilaya está constituida, a su vez, por una serie de comunas que son las colectividades territoriales básicas.
} 


\section{Una nueva oportunidad: el pluralismo político}

El fin de la década de los ochenta vendrá marcado por una cierta apertura. Una serie de protestas y manifestaciones populares recurrentes acaban desembocando en las fuertes revueltas sociales de 1988, que ponen de manifiesto el desencanto de la población ante la deriva política y económica del país (Stora, 2004: 98-101). El gobierno de Chadli Bendjedid se verá abocado a adoptar una nueva Constitución en $1989^{\circ}$, que, como novedad, pondrá fin al régimen institucional de partido único al reconocer, en el artículo 40, el derecho a crear "asociaciones de carácter político" lo que contribuirá a crear un contexto más favorable para incentivar la participación femenina al ampliarse el abanico de opciones políticas. A partir de esta fecha se desarrolló un importante movimiento de mujeres canalizado a través de tres tipos de asociaciones: moderadas, preocupadas por conservar las raíces arabo-musulmanas del pueblo argelino; laicas, más próximas al pensamiento feminista occidental; y, asociaciones islamistas, influenciadas por el reformismo musulmán (Pérez Beltrán, 1998: 91-94).

Sin embargo, en estos momentos de relativa apertura, el escenario político dará un vuelco inesperado. En las primeras elecciones pluralistas, celebradas en junio de 1990, para renovar las asambleas locales y, en los comicios legislativos de diciembre de 1991, los islamistas del Frente Islámico de Salvación (FIS) obtuvieron la mayoría. La intervención del ejército desembocó en el período conocido como la década negra, marcada por la violencia y los atentados (Addi, 2006: 3). Tras el golpe de estado el proceso electoral fue interrumpido y se declaró el estado de urgencia. Las mujeres sufrirán este conflicto con especial crudeza y la lucha por la propia vida ${ }^{9}$ dejó en segundo plano la reivindicación de los derechos femeninos. No obstante, la dinámica asociativa femenina no se paralizó y surgieron nuevas organizaciones de mujeres muy politizadas por el contexto que se centraron casi exclusivamente en la erradicación del islamismo por considerarlo responsable de la caótica situación del país (Pérez Beltrán, 1996: 206-207). Asimismo, en este período 12 mujeres participaron en el Consejo Nacional de Transición, que se mantuvo en funcionamiento entre los años 1994 y 1997, llegando una de ellas incluso a ostentar la presidencia de la comisión (Saad-Zoy et al, 2010: 38).

Durante estos convulsos años, "como primera medida para reactivar la normalización política e institucional" (Pérez Beltrán, 2017: 265), fue promulgada la Constitución de $1996^{10}$ que, a diferencia del anterior texto constitucional que hablaba de "asociaciones de carácter político", reconoció explícitamente y garantizó el derecho a crear partidos políticos (art. 42). En este marco también fue promulgada la Ley Orgánica relativa a los partidos políticos ${ }^{11}$ (Pérez Beltrán, 2017: 265) y por primera vez el poder legislativo pasó a ser ejercido por un Parlamento compuesto por dos cámaras, la Asamblea Popular Nacional y el Consejo de la Nación (art. 98).

Este mismo año, tiene lugar un relevante acontecimiento político de cara a los derechos femeninos. En 1996, Argelia ratificó ${ }^{12}$ la Convención sobre la Eliminación de Todas las Formas de Discriminación contra la Mujer (CEDAW, según sus siglas en inglés). Esta convención, adoptada en 1979 por la

\footnotetext{
${ }^{8}$ http://www.conseil-constitutionnel.dz/index.php/fr/1989 [consulta: 26 de octubre de 2020].

9 Numerosas mujeres fueron víctimas de la violencia islamista, pero también de la de las fuerzas de seguridad institucionales. La militante feminista Nabila Djahnine, presidenta de la asociación Tighri n'Tmettut (Grito de mujer) y miembro del Movimiento Cultural Bereber fue asesinada por los islamistas (Pérez Beltrán, 1996: 215).

${ }^{10}$ https://mip.univ-perp.fr/constit/dz1996c.htm [consulta 26 de octubre 2020].

11 Orden 97-07 de 6 de marzo de 1997, JORA n. 12, de 6 de marzo de 1997.

12 Decreto Presidencial n. 96-51 de 22 de enero, JORA n. 6 de 24 de enero.
} 
Asamblea General de Naciones Unidas, y en vigor desde 1981, continúa siendo a día de hoy la más importante en materia de igualdad de sexos. Precisamente, en el ámbito político, el artículo 4 de la Convención (CEDAW), y las resoluciones de la Asamblea General de las Naciones Unidas de 2003 (art. 1) y de 2011 (art. 9) sobre la participación de la mujer en la política ${ }^{13}$ propondrán la adopción por los Estados de medidas transitorias de discriminación positiva con el fin de lograr una representación paritaria de mujeres y hombres.

Paradójicamente, la trascendencia de la ratificación de este tratado se verá eclipsada por la posibilidad, a la que se acoge Argelia, de formular reservas que de facto van en contra de la propia naturaleza del tratado.

Todos estos pasos no serán suficientes para provocar cambios inmediatos en el espectro político argelino y el gobierno tardará todavía unos años en adoptar una medida concreta que impulse una mayor participación de las mujeres. Prueba de ello son los datos procedentes de los comicios a la APN de 1997 donde las mujeres sólo alcanzaron una representatividad del 3,3\%, con 13 diputadas (CIDDEF, 2016: 69). Este dato contrasta, no obstante, con su alto nivel de participación como electoras, ya que las mujeres que ejercieron su derecho al voto supusieron el 46,59\% del electorado, lo que revela un interés cada vez mayor por la política, a pesar de la vigencia de prácticas como el voto delegado que constituía una "aberración en la historia política moderna de Argelia" ya que suponía la marginalización del voto de las mujeres que era ejercido por los miembros masculinos de su familia (Saad-Zoy et al, 2010: 37).

La Ley electoral de $1989^{14}$ regulaba el voto por procuración que se ejercía, cumpliendo una serie de requisitos formales, a petición de un elector que se encontrase en una serie de supuestos tasados. Sin embargo, en el caso de los cónyuges, ni siquiera era necesario contar con un documento de procuración formal ya que el artículo 53 de la citada ley dispensaba de esta formalidad al cónyuge que pudiera justificar en el momento del voto su vínculo matrimonial mediante la mera presentación del libro de familia. Con esta dispensa, utilizada abusivamente, y considerada por las feministas como un atentado a la ciudadanía, se excluía de facto a las mujeres del derecho al ejercicio del voto (Benghabrit-Remaoun, 2012: 137). La Asociación Independiente para el Triunfo de los Derechos de las Mujeres (AITDF), centrada en reivindicar los derechos políticos de las argelinas, se oponía frontalmente a esta práctica y junto a otras asociaciones feministas crearon, en octubre de 1990, un Comité Nacional de Lucha ${ }^{15}$ contra los artículos 53 y 54 de la Ley Electoral (Pérez Beltrán, 1997: 323-324). Finalmente, aunque bastante más tarde, las presiones surtieron efecto y el 28 de octubre de 1998 la coordinación entre los movimientos feministas consiguió que el Consejo Constitucional declarase la inconstitucionalidad de esta práctica (ibid.: 331).

A partir del año 2000 la determinación del gobierno para llevar a cabo una política favorable a las mujeres se vio reflejada en la creación de una serie de instancias oficiales encargadas de mejorar la condición femenina aunque limitadas a aspectos muy puntuales, dando lugar a una apariencia de "feminismo de Estado" (Benghabrit-Remaoun, 2012: 138), que seguía, no obstante, sin cuestionarse la vigencia del Código de familia, texto que, además de discriminatorio, constituía una anomalía al estar situado "fuera del ordenamiento jurídico" y reenviar directamente a la ley islámica en caso de laguna legal (Graba, 2013: 5).

En el terreno político, se observa como en las posteriores elecciones, el número de diputadas electas irá ascendiendo progresivamente, aunque todavía la brecha con respecto al número de diputados seguirá siendo considerable. En la legislatura comprendida entre los años 2002 y 2006 las candidatas electas a la APN supondrán un $6,9 \%$ de los diputados con 27 mujeres electas, mientras que en el

\footnotetext{
${ }^{13} \mathrm{~A} / \mathrm{RES} / 58 / 142$ de 22 de diciembre de 2003 y A/RES/66/130 de 19 de diciembre de 2011.

${ }^{14}$ Ley 89-13 de 7 de agosto de 1989, Ley Electoral General (arts. 50-60).

${ }^{15}$ Este Comité, además de integrar a las asociaciones feministas más importantes, incluía algunos partidos políticos como el Frente de Fuerzas Socialistas (FFS), Partido Socialista de los Trabajadores (PST), Partido de los Trabajadores (PT) y Agrupación por la Cultura y la Democracia (RCD), entre otras organizaciones (Pérez Beltrán, 1997: 331).
} 
período comprendido entre 2007 y 2012 el porcentaje de diputadas ascenderá hasta el 7,7\%, con 30 mujeres:

[Fig. 4] Resultados por géneros de las elecciones nacionales celebradas entre 1997 y 2007.

\begin{tabular}{|l|c|c|c|}
\hline ELECCIONES APN & $1997-2002$ & $2002-2006$ & $2007-2012$ \\
\hline Diputados & 376 & 362 & 359 \\
\hline Diputadas & 13 & 27 & 30 \\
\hline Total escaños & 389 & 389 & 389 \\
\hline \multicolumn{1}{|c|}{$\%$ Mujeres } & $3,3 \%$ & $6,9 \%$ & $7,7 \%$ \\
\hline
\end{tabular}

Fuente: elaboración propia con los datos procedentes de CIDDEF (2016: 69)

La presencia marginal de mujeres electas en el parlamento guarda una estrecha relación con su escasa presencia en las listas electorales de los partidos políticos. En las elecciones de 1997 se presentaron 322 mujeres, el 4,15\% de un total de 7427 candidatos, y en las elecciones de 2002, nos encontramos con 694 mujeres, el 6,9\%, de 9358 candidatos (Benghabrit-Remaoun, 2012: 142-143).

La misma tendencia se observa a nivel de asambleas locales, donde la representación femenina es muy escasa, y donde las candidatas que resultan elegidas no acceden a las funciones de decisión política y administrativa (Gribaa, 2009: 19). En los comicios de 1997, en las APW sólo resultaron elegidas 62 mujeres de 905 candidatos, aunque en este nivel posteriormente se producirá un ligero repunte y, en 2002, el número de mujeres electas en las asambleas de wilaya será de 113 . En cuanto a las elecciones a las APC, en 1997 resultaron elegidas 75 mujeres de 1281 candidatos, ascendiendo a 147 en las elecciones de 2002 de un total de 3679 candidatos:

[Fig. 5] Proporción de candidatas electas en las elecciones locales de 1997 y 2002.

\begin{tabular}{|l|c|c|c|c|}
\hline & \multicolumn{2}{c|}{1997} & \multicolumn{2}{c|}{2002} \\
& $\begin{array}{c}\text { Candidatos } \\
\text { Totales }\end{array}$ & $\begin{array}{c}\text { Candidatas } \\
\text { electas }\end{array}$ & $\begin{array}{c}\text { Candidatos } \\
\text { totales }\end{array}$ & $\begin{array}{c}\text { Candidatas } \\
\text { electas }\end{array}$ \\
\hline APC & 1281 & 75 & 3679 & 147 \\
\hline APW & 905 & 62 & 2684 & 113 \\
\hline \multicolumn{2}{|c|}{ Fuente: elaboración propia con los datos de Gribaa (2009: 18) }
\end{tabular}

Ante esta falta de avances en el compromiso de las mujeres con la política, representantes de partidos políticos y colectivos como el Centro de Información y Documentación sobre los Derechos del Niño y de la Mujer (CIDDEF), pusieron en marcha una serie de iniciativas, destacando la creación, en 2005 , de una coalición de mujeres encargadas de presentar un alegato en pro de la promulgación de una futura ley de cuotas. 
Las demandas de la sociedad civil, junto a la necesidad cumplir con los compromisos adquiridos al ratificar la CEDAW llevarán a que, con ocasión de la revisión constitucional de $2008^{16}$, se añada el artículo 31 bis $^{17}$ que establecía que el Estado trabajaría "en la promoción de los derechos políticos de la mujer aumentando sus oportunidades de acceso a la representación en las asambleas electas". Las modalidades de aplicación de este artículo se determinarían en una ley que se hizo esperar hasta 2012, con la promulgación de la Ley Orgánica que fija las modalidades para aumentar las posibilidades de acceso de la mujer a la representación en las asambleas electas.

\section{Las cuotas de género: definición y tipología}

Antes de entrar en el análisis específico de la Ley de cuotas adoptada en Argelia, conveniente realizar una serie de precisiones teóricas acerca del mecanismo de las cuotas y de sus diferentes modalidades.

En política, una cuota se define como una "medida de acción afirmativa que establece un porcentaje o un número para la representación de un grupo específico, en este caso mujeres, bajo la forma más frecuente de un porcentaje mínimo requerido" (Dahlerup, 2007: 78). Las cuotas constituyen una acción de discriminación positiva o "discriminación inversa que provocaría un daño en los colectivos no beneficiados por la medida, en este caso, los hombres (reverse discrimination)" (Uribe Otalora, 2013: 159).

Las cuotas de género constituyen una de las medidas más extendidos para tratar alcanzar el equilibrio de género en las instituciones políticas. Actualmente, en torno a la mitad de los países del mundo aplican algún tipo de cuota electoral de género (Dahlerup, 2007: 78). Los tratados internacionales como la CEDAW consideran que las cuotas género no son un fin en sí mismas, sino que constituyen medidas de carácter transitorio hasta que se alcance la igualdad, aunque, de facto, en la mayoría de los países que han implementado este sistema han seguido vigentes en el tiempo, por las dificultades que experimentan las mujeres para conseguir situarse al mismo nivel que los hombres.

Existen diferentes tipos de cuotas de género en política, aunque los más frecuentes en la actualidad son (IDEA, 2020):
a) Cuota de escaños reservados
b) Cuotas legales de candidatos
c) Cuotas de los partidos políticos

Las cuotas de escaños reservados regulan el número de mujeres electas y aseguran la representación de las mujeres en las asambleas. Se trata de una medida rápida y efectiva especialmente en países marcadamente patriarcales. Las cuotas legales de candidatos y las cuotas de los partidos políticos establecen un porcentaje mínimo de mujeres en las listas de candidatos ya sea como requisito legal, en el primer caso, o como medida incluida voluntariamente en los estatutos de los distintos partidos políticos, en el segundo caso (Dahlerup, 2009). Solo el sistema de cuota de escaños reservados garantiza unos resultados que se traducen en una cierta representación a las mujeres (Dahlerup, 2007: 83). Los otros dos tipos de cuotas mencionados no sólo no garantizan la representación de las mujeres, sino que además deben ir acompañados de medidas adicionales a la hora de elaborar las listas de candidatos que impidan que las mujeres sean relegadas a los últimos puestos. Por ello, en algunos países como Bélgica o Argentina se establece

\footnotetext{
${ }^{16}$ Ley n. 08-19 de 15 noviembre 2008, JORA n. 13 de 16 de noviembre.

${ }^{17}$ Art. 35, en la Constitución de 2016.
} 
un sistema de "doble cuota" en el que además de garantizarse un porcentaje mínimo de mujeres en las listas electorales se establecen una serie de requisitos acerca del orden de posición de los candidatos en las listas para evitar que las candidatas pierdan finalmente sus posibilidades reales de resultar elegidas (IDEA, 2020). Una medida, en este sentido, consiste en recurrir a la utilización de "listas cremallera" en las que la alternancia "hombre-mujer-hombre" o bien "mujer-hombremujer" garantizan una mayor presencia de mujeres (Uribe Otalora, 2013: 188).

Los detractores de las cuotas electorales de género consideran que se trata de una medida "intervencionista y antidemocrática de imagen o maquillaje" (Uribe Otalora, 2013: 188) que resulta discriminatoria al suponer "una violación del principio liberal del mérito", mientras que los partidarios del sistema ven las cuotas como una medida basada en la equidad que funciona como "una compensación frente a la discriminación, directa o estructural" que sufren las mujeres (Dahlerup, 2007: 73).

La opción por una determinada cuota de género suscita además un debate en torno a dos conceptos de igualdad: igualdad de oportunidades e igualdad de resultados, estrechamente relacionados con los distintos modelos de sociedad en los que se aplican estos sistemas. La crítica feminista a menudo considera que la igualdad de oportunidades no basta ya que no es "real" puesto que rara vez se produce entre hombre y mujeres, por la posición privilegiada que ocupan los primeros. Por ello, están a favor de implementar medidas para conseguir una igualdad de resultados que los liberales, con una concepción individualista de la sociedad, consideran injusta (Dahlerup, 2007:74). Esta igualdad de resultados no deja de ser en el fondo una igualdad cuantitativa que no presupone que a nivel político las mujeres estén en la misma situación ni al mismo nivel que los hombres. Las cuotas de escaños reservados, en este sentido, son las que garantizan un resultado mientras que las cuotas de candidatos no siempre cumplen con las expectativas depositadas en ellas.

Para que la cuota sea efectiva debe ser una cuota "legal", es decir, que venga impuesta por una norma que le otorgue carácter obligatorio y cuyo incumplimiento lleve aparejado algún tipo de sanción o penalización. De otro modo se dejaría en las manos de los partidos políticos la decisión de aplicarla. Por ello, la paridad electoral no depende solo de la aplicación de una cuota, sino que intervienen otros factores como la voluntad del legislador al definirla, el tipo de sistema y la predisposición de los propios partidos políticos (Uribe Otalora, 2013: 188).

La naturaleza del sistema electoral puede influir también a la hora de implementar las cuotas de modo que para algunos autores como Dahlerup (2009) resulta más fácil su introducción en los sistemas de representación proporcional, aunque también se han implementado cuotas en sistemas mayoritarios. Lo cierto es que no existe un consenso entre la comunidad científica que determine qué sistemas electorales -proporcionales o mayoritarios- favorecen más la igualdad entre hombres y mujeres (Uribe Otalora, 2013: 185).

Los efectos de la introducción de un determinado sistema de cuotas deben ser estudiados tanto cuantitativa como cualitativamente. En este trabajo nos centramos básicamente en el aspecto cuantitativo, es decir, en el impacto de la medida sobre el número de candidatas electas en los diferentes comicios. No obstante, también sería interesante, aunque excede el ámbito de este análisis, conocer el impacto cualitativo de las cuotas, es decir, en qué aspectos ha influido positivamente una mayor presencia femenina y si ha tenido algún impacto en la adopción de políticas y leyes más favorables a la igualdad. 
En países norteafricanos próximos, cultural y geográficamente como Marruecos, también se han implementado sistemas de cuotas para aumentar la representatividad femenina en el campo político. En el caso de Marruecos se optó por una cuota de género de escaños reservados que son elegidos mediante un sistema electoral de representación proporcional con listas electorales nacionales y cerradas. En este caso, la ley establece ${ }^{18}$ que se reservarán 60 escaños de la Cámara Baja a las mujeres, así como otros 30 que irán a parar a jóvenes -hombres y mujeres- de menos de 40 años (IDEA, 2020). A nivel subnacional, la ley ${ }^{19}$ marroquí establece una cuota de mujeres en función de la cual al menos un tercio de los escaños de los consejos regionales deberá ser reservado para las mujeres (ibidem.). El caso de Túnez, al igual que en Argelia, se adoptó el sistema de cuotas de candidatos legisladas aplicables en un sistema de representación electoral proporcional. Sin embargo, difiere del sistema argelino a la hora de determinar los porcentajes reservados a ambos géneros ya que la ley ${ }^{20}$ tunecina establece que en las listas a la Asamblea de representantes del pueblo se respetará necesariamente la paridad entre hombres y mujeres. Además, los candidatos se colocarán en las listas alternando candidatos masculinos y femeninos. A nivel de elecciones municipales y regionales también se respetará la igualdad (50-50\%) entre hombres y mujeres, no aceptándose ninguna lista que incumpla este principio ${ }^{21}$ (IDEA, 2020).

\section{El camino hacia la ley de cuotas argelina}

En Argelia el proceso de adopción de la ley de cuotas fue largo y complejo. Hicieron falta más de dos años de negociaciones desde el inicio de los trabajos de la comisión, creada al efecto en marzo de 2009, para que el texto definitivo viese la luz.

En este camino la sociedad civil, ha jugado un importante papel transformador y de concienciación, especialmente gracias a la labor desarrollada por los movimientos y asociaciones feministas que han ejercido presiones para impulsar cambios en las políticas públicas (Thieux, 2018: 87).

Finalmente, el proyecto de ley orgánica fue adoptado en el seno del Consejo de Ministros en agosto de 2011 tras introducir una primera restricción. El número de candidatas en las listas electorales no podría ser inferior al 30\%, tal y como habían reclamado las asociaciones de mujeres, pero, y aquí estaba el cambio, esta proporción se impuso únicamente a las comunas con más de 20000 habitantes "ante el temor de no encontrar candidatas en las pequeñas comunas debido a la impronta de las costumbres y las tradiciones" (Benzenine, 2013: 6). Esta precisión, lejos de beneficiar a las mujeres contribuía precisamente a lo contrario, es decir, a perpetuar estereotipos que relegan a la mujer a un rol determinado apartado de la vida pública.

El proyecto de ley pasará a discutirse en el Parlamento donde la deliberación será mucho más ardua y pondrá de manifiesto las tremendas divergencias existentes entre los distintos partidos. Por un lado, estaban los partidos que rechazaban abiertamente la medida, principalmente algunos partidos islamistas como Ennahda ( $M N)$, que alegaba que "una decisión que no tuviera en cuenta el nivel cultural y las mentalidades de la sociedad no podría ser práctica" pero, también el Partido de los Trabajadores $^{22}$ que se oponía a la medida por considerar que para conseguir una representación

\footnotetext{
${ }^{18}$ Ley Orgánica n. 20-16 de 2016 modificando y completando la Ley Orgánica n. 27-11 de 2011 relativa a la Cámara de representantes (art. 23, 2).

${ }^{19}$ Ley Orgánica n. 59-11 de 2011 relativa a la elección de los miembros de los consejos colectivos territoriales (arts. 76 y 77).

${ }^{20}$ Ley Orgánica n. 16 de 26 de mayo de 2014 relativa a las elecciones y a los referéndums (art. 24).

${ }^{21}$ Ley Orgánica n. 7 de 14 de febrero de 2017 (art. 49, 9), que revisa y complete la Ley Orgánica n. 16 de 26 de mayo de 2014 relativa a las elecciones y a los referéndums.

${ }^{22}$ Partido de izquierda de orientación trotskista..
} 
femenina de calidad antes había que adoptar medidas como la derogación del Código de la familia (Benghabrit- Remaoun, 2012: 159).

El Frente Nacional Argelino (FNA), de orientación de derechas, tampoco era favorable a las cuotas por considerar que su aplicación no sería posible al no existir control de los partidos por parte del Estado, en cuyo caso sería mejor impulsar la participación de la mujer en la administración, opinión que también compartía el RCD favorable a impulsar la presencia de mujeres en el gobierno ${ }^{23}$. Otros partidos como la Reagrupación Nacional Democrática (RND), de centro derecha y próximo al FLN, el propio FLN y el Movimiento de la Sociedad por la Paz (MSP) ${ }^{24}$ se mostraban favorables a incrementar el número de mujeres en política, aunque diferían en cuanto al porcentaje mínimo de candidatas exigible en las listas (Benghabrit-Remaoun, 2012: 139). Además, el MSP, aunque no se oponía a la medida, hacía público su temor de que se diera prioridad a las nominaciones femeninas en detrimento de la competencia ${ }^{25}$.

Finalmente, la ley votada el 3 de noviembre en vía parlamentaria acabará defraudando las expectativas depositadas en el proyecto inicial del gobierno. El motivo de la discordia resultó ser la cuota mínima del 30\% de candidatas. La ley votada en fase parlamentaria rebajó esta proporción al $20 \%$ cuando se tratase de wilayas en las que el número de escaños a la APN fuera igual a cuatro. Por lo tanto, la tasa de representatividad de las mujeres no será homogénea, sino que variará en función de criterios territoriales. Algunas parlamentarias manifestaron públicamente su desilusión como ponen de manifiesto las palabras de Halima Lakehal, diputada del FLN, al señalar que "el texto del presidente se había vaciado de sentido", ya que si él mismo "había querido imponer una cuota del 30\% era porque conocía la mentalidad de los hombres" (Dubruelh, 2011) y en el mismo sentido, de Amina Gharbi Bounab, también del FLN, quien consideró que la ley resultante constituía "una discriminación territorial y sexual en contra de las mujeres" (ibid.).

La ley orgánica 12-03, que fija las modalidades para aumentar las posibilidades de acceso de la mujer a la representación en las asambleas electas ${ }^{26}$, fue promulgada el 12 de enero de 2012, como parte del proceso de renovación política emprendida en esos momentos por el gobierno de Bouteflika. El texto vio la luz en el contexto de las primaveras árabes, movimiento que no llegó a eclosionar en Argelia del mismo modo que en países vecinos a pesar de la existencia de fuertes tensiones sociales y del descontento con la clase política. La debilidad y fragmentación del tejido social, así como la falta de formaciones políticas verdaderamente independientes del régimen de Bouteflika produjeron la rápida desarticulación de las movilizaciones (Pérez Beltrán, 2017: 269).

Sin embargo, las protestas provocaron la pronta respuesta del gobierno que se apresuró a levantar el estado de urgencia, vigente desde 1992, a convocar unos nuevos comicios, y a promulgar una serie de leyes relacionadas con las libertades públicas que pretendían "simbolizar el nuevo talante aperturista" del régimen (Pérez Beltrán, 2017: 270). Además de la Ley de cuotas también fue promulgada la Ley Orgánica relativa a los partidos políticos ${ }^{27}$ que incluía en su articulado varias referencias a la representatividad de las mujeres en los órganos de dirección y gestión de los partidos (Pérez Beltrán, 2016: 77). En concreto, el art. 17 establece que "entre los miembros

\footnotetext{
23 "Projet de loi imposant un quota de 33\% de femmes sur les listes électorales", Algeria-Watch (publicado en EI Watan el 20 de septiembre de 2011 [ consulta: 30 de octubre de 2020].

${ }^{24}$ Partido islamista, próximo a los Hermanos Musulmanes.

25 "Projet de loi imposant un quota de 33\% de femmes sur les listes électorales", op. cit.

${ }^{26}$ JORA, n. 1 de 14 de enero de 2012, p. 39.

${ }^{27}$ Ley Orgánica n. 12-04 de 12 de enero de 2012 relativa a los partidos políticos, JORA de 15 de enero de 2012.
} 
fundadores" debe existir una "proporción representativa" de mujeres, y la misma expresión se vuelve a emplear en el art. 24 referido al congreso constitutivo del partido. Por su parte, el art. 41 determinaba que todo partido político debería "incluir un porcentaje de mujeres dentro de sus órganos directivos" (Pérez Beltrán, 2017: 276) aunque no se especificaba ni el porcentaje ni la forma de conseguir estos objetivos, con lo cual se tratan más bien de "declaraciones de intenciones" (Pérez Beltrán, 2016: 77).

En cuanto a la Ley de cuotas, el texto resultante es bastante breve y está compuesto únicamente de 8 artículos, pero, el hecho de haber sido promulgado con la forma de ley orgánica, lo coloca por encima de las leyes ordinarias, lo que da idea de la relevancia concedida al tema de fondo. El tipo de cuota de género que establece esta ley es el de cuota legislada y obligatoria de candidatos en las listas de los partidos políticos.

El artículo 2 constituye el núcleo central del texto puesto que es el que establece las cuotas mínimas reservadas a las mujeres estipulando que:

“El número de mujeres que figuren en las listas de candidatura, ya sean independientes o presentadas por uno o varios partidos políticos, no debe ser inferior a las proporciones definidas a continuación, proporcionalmente al número de escaños al poder.

\section{Elecciones a la Asamblea Popular Nacional:}

$-20 \%$ cuando el número de escaños sea igual a 4 ,

$-30 \%$ cuando el número de escaños sea igual o superior a 5 ,

-35\% cuando el número de escaños sea igual o superior a 14,

$-40 \%$ cuando el número de escaños sea igual o superior a 32 ,

$-50 \%$ para los escaños de la comunidad nacional al extranjero.

\section{Elecciones a las asambleas populares de wilayas:}

$-30 \%$ cuando el número de escaños es de 35, 39, 43 y 47 escaños (es decir, 10, 12, 13 y 14 mujeres, respectivamente)

$-35 \%$ cuando el número de escaños es de 51 a 55 escaños (18 hasta 53 escaños y 19 mujeres si hay 54 o 55 escaños).

\section{Elecciones a las asambleas populares comunales:}

$-30 \%$ para las asambleas populares comunales situadas en las capitales de las daïras ${ }^{28}$ y en el seno de comunas cuyo número de habitantes sea superior a veinte mil (20.000)".

\footnotetext{
${ }^{28}$ La daïra es una subdivisión administrativa de la wilaya que reagrupa a varias comunas.
} 
El artículo 3 especifica que "Ios escaños serán repartidos en función del número de votos obtenidos por cada lista", teniendo que respetarse las proporciones fijadas en el artículo precedente que están "obligatoriamente reservadas a las candidatas femeninas según su clasificación nominal en las listas.

Para tratar de evitar posibles confusiones o incumplimientos a la hora de presentar las candidaturas, el artículo 4 ordena expresamente que "se mencione el sexo del candidato" mientras que el artículo 5 fija el plazo para adecuar las listas a lo previsto en el artículo 2 sin que dicho plazo pueda exceder del mes anterior a las elecciones, añadiendo, como única medida de presión para hacer cumplir la ley, que "toda lista de candidatura a unas elecciones constituida violando las prescripciones del artículo 2 de la presente ley orgánica será rechaza". Aparte de este rechazo no se prevé ningún otro tipo de sanción al partido político incumplidor que pudiera resultar más efectiva. En su lugar, el artículo 7 ofrece un incentivo, que será determinado por vía reglamentaria, para fomentar la inclusión de mujeres en las listas ya que establece que "los partidos políticos se podrán beneficiar de una ayuda financiera específica del Estado, según en número de sus candidatas que hayan resultado electas en las asambleas populares comunales, en las wilayas y en el Parlamento", incentivo discutible a no ser que se traduzca en una mejora de la situación de las mujeres en los posibles partidos beneficiarios.

Por último, el artículo 6 resuelve la cuestión del reemplazo de un candidato o de un miembro electo conforme a lo previsto en la Ley Orgánica de régimen electoral o en las leyes relativas a las comunas o a las wilayas, añadiendo el único requisito de que el sustituto tenga el mismo sexo que tuviera la persona a reemplazar.

Las listas de candidatos a las APW y a las APC deberán estar formadas por tantos candidatos como escaños correspondan a cada circunscripción y además deberán contar con un número determinado de suplentes que no puede ser inferior en cada caso al 30\% de los escaños en juego.

\section{Efectos y desafíos de la entrada en vigor de la ley de cuotas}

La Ley de cuotas se aplicó por primera vez con ocasión de las elecciones legislativas de 10 de mayo de 2012. En estos comicios la participación global del electorado fue del $43,14 \%$, destacando el alto número -casi dos millones- de votos nulos o en blanco, que algunos autores interpretaron como un rechazo ciudadano a la oferta política, "expresado de forma diferente a la abstención" (Saïfi y Panzeri, 2012: 6). Aun así, la participación se incrementó con respecto a las elecciones anteriores de 2007 en las que se alcanzó una abstención récord al concurrir a las urnas solamente el 35,5\% el electorado ${ }^{29}$ poniendo de manifiesto la profunda crisis de confianza ciudadana en la política como vía para solucionar los problemas del país.

Los comicios de 2012 supusieron un punto de inflexión ya que por primera vez el porcentaje de mujeres electas en la APN alcanzará el 31\%, casi un tercio de los 462 escaños, resultando elegidas 143 diputadas, es decir 113 parlamentarias más que en las elecciones de 2007 (Saïfi y Panzeri, 2012: 6). Estos resultados colocarán a Argelia por primera vez a la cabeza de los países árabes en cuanto a participación femenina en política (Benzenine, 2013: 1).

\footnotetext{
${ }^{29}$ Véase: Dris-Aït Hamadouche (2009).
} 
[Fig. 6] Resultados de las elecciones legislativas a la APN en el período 2007-2020.

\begin{tabular}{|l|l|l|l|}
\hline $\begin{array}{l}\text { LEGISLATURA } \\
\text { APN }\end{array}$ & $2007-2012$ & $2012-2016$ & $2017-2020$ \\
\hline Diputados & 359 & 331 & 344 \\
\hline Diputadas & 30 & 143 & 118 \\
\hline Total escaños & 389 & 474 & 462 \\
\hline \% Mujeres & $7,7 \%$ & $30,2 \%$ & $25,5 \%$ \\
\hline
\end{tabular}

Fuente: elaboración propia con los datos procedentes de CIDDEF (2016: 68)

Paradójicamente, estos primeros éxitos no conseguirán mantener la tendencia al alza en cuanto a participación de las mujeres como reveló el descenso del número de diputadas en los posteriores comicios de 2017, en donde pasaron a ocupar 118 escaños, bajando el porcentaje de mujeres electas al $25,5 \%$. Ni siquiera el hecho de que el número de mujeres votantes hubiese superado al número de hombres contribuyó a mejorar la situación ${ }^{30}$.

La mayor parte de las diputadas electas a la Asamblea en esta ocasión militaban en las filas del FLN, pasando a ocupar 51 de los 166 escaños obtenidos por esta formación política. El segundo partido con mejores resultados fue la Reagrupación Nacional Democrática (RND), aliado tradicional del FLN, que obtuvo 101 escaños, de los cuales 33 fueron a parar a las diputadas. Estos dos partidos ostentaban la mayoría en la APN, consolidando de nuevo su posición al frente de un gobierno en el que el poder ejecutivo goza de preeminencia. Por ello, en cierto modo, la introducción de las cuotas, en tanto que medida impulsada directamente desde el poder, contribuye en el fondo a seguir manteniendo un statu quo muy cuestionado por el electorado ${ }^{31}$.

El mecanismo de las cuotas también se ha aplicado en los comicios locales, nivel en el que resulta fundamental llevar a cabo una labor previa de concienciación con el objetivo de atraer a las mujeres a la política, especialmente en comunidades remotas muy conservadoras. Es precisamente en este nivel donde se deciden realmente los asuntos que más efectos pueden tener sobre la vida cotidiana de las propias mujeres.

En las elecciones locales celebradas el 23 de noviembre de 2017, el FLN, pese a ser la fuerza más votada, sufrió un importante retroceso con respecto a los resultados de $2012^{32}$. De las 1540 comunas del país, el FLN pasó a controlar 603 y perdió 400. Pese a este importante revés del partido mayoritario, de un total de los 24876 puestos, las mujeres obtuvieron 4132, lo que se traduce en una tasa de representatividad del $16,6 \%$ en las Asambleas Populares Comunales (APC), una cifra

\footnotetext{
${ }^{30}$ http://www.interieur.gov.dz/index.php/fr/le-ministere/le-minist\%C3\%A8re/communiques/202communiqu\%C3\%A9s-d\%C3\%A9clarations/1681-fiche-statistique-des-\%C3\%A9lections-l\%C3\%A9gislatives-2017.html [consulta: 26 de octubre de 2020].

31 Estos comicios legislativos fueron los últimos celebrados antes de las controvertidas presidenciales de 2019 , desarrolladas en un clima de movilizaciones y malestar generalizado de la población a raíz del intento fallido de Bouteflika de volver a presentar su quinta candidatura, que desembocaron en su dimisión (Bustos, 2019).

${ }^{32}$ http://www.interieur.gov.dz/index.php/fr/actualit\%C3\%A9s/1850-r\%C3\%A9sultats-des-\%C3\%A9lections-locales-de2012.html [consulta: 26 de octubre de 2020].
} 
significativa teniendo en cuenta las dificultades de algunos partidos para encontrar candidatas a nivel local ${ }^{33}$. Asimismo, en las APW, la representación política femenina llegó hasta el $29,7 \%$, quedando ya lejos de datos tan bajos como los del 2007, con sólo un $0,76 \%$ de mujeres en las APC y un $6,9 \%$ en las $\mathrm{APW}^{34}$.

Estos resultados llevaron a que, en 2018, el Foro Económico Mundial, situará a Argelia en cuanto a participación de las mujeres en política, en el puesto 85 de la clasificación que mide la brecha de género, conocida como Global Gender Gap (WEFORUM, 2018: 11), colocándose por encima de Marruecos, que ocupaba el puesto 102, pero por debajo de Túnez, que ostentaba el puesto 55.

Sin embargo, a pesar de los avances, la puesta en marcha de esta ley se enfrenta aún a una serie de desafíos que contribuyan a impulsar definitivamente a las mujeres en el terreno político. La medida adoptada se revela insuficientes para alcanzar la realización efectiva del principio de igualdad.

En primer lugar, el propio ámbito de aplicación de la ley, limitado únicamente a las candidaturas que se presenten a la APN y las APW y APC deja fuera otras instancias de poder político como la segunda cámara del parlamento, el Consejo de la Nación, cuyos miembros son elegidos, dos tercios por sufragio indirecto y secreto entre los miembros de las APW y de las APC y el tercio restante es designado directamente por el presidente de la República.

En segundo lugar, la ley argelina, a diferencia de otras legislaciones como la tunecina no establece la paridad entre hombres y mujeres al fijar las cuotas. Es más, las cuotas establecidas no toman en cuenta datos demográficos para realizar un reparto equilibrado de las candidaturas, sino que, en su lugar, establece unos porcentajes mínimos diferentes en función del número de habitantes o de los escaños a repartir en las distintas circunscripciones dando lugar finalmente a una discriminación por razones geográficas.

En tercer lugar, el único criterio que menciona la ley en cuanto a la forma de inscribir a los candidatos en las listas es la "clasificación nominativa", es decir, alfabética, que no garantiza la visibilización de las candidatas ni que éstas tengan finalmente opciones de ser elegidas. Este problema se ve agravado en circunscripciones pequeñas con un bajo número de escaños a repartir en las que los hombres suelen colocarse por delante de las mujeres que acaban siendo relegadas de la vida política. Por ello, lo recomendable habría sido recurrir a un sistema de "listas cremallera", que en un sistema electoral proporcional como el argelino es el más idóneo para alcanzar la paridad en la representación femenina (Benzenine, 2013: 11).

En cuarto lugar, la ley se dirige exclusivamente a los partidos políticos, a los que impone el deber de contribuir a reforzar la representación de las mujeres en las asambleas electas mediante su inclusión en las listas de las candidaturas electorales. Esta responsabilidad acarrea tensiones internas. Los partidos, pasan así a ser los depositarios de una obligación que el propio Estado había asumido en la Constitución y que no ha estado acompañada de ninguna otra medida en paralelo orientada a mejorar el papel de las mujeres en el seno de estos partidos o a potencias su afiliación a los mismos. Por ello, si las cuotas no se ven reforzadas con otras estrategias, existe el riesgo de que la elección de candidatas no se ajuste a los prescrito. Puede suceder que los partidos incluyan a las candidatas en sus listas únicamente en función de su sexo y no de su idoneidad ni de su capacitación para tomar

\footnotetext{
${ }^{33}$ http://www.interieur.gov.dz/index.php/fr/actualit\%C3\%A9s/2028-r\%C3\%A9sultats-elections-locales-du-23novembre-2017.html [consulta: 14 de junio de 2020].

$34 \quad$ https://www .lemonde.fr/afrique/article/2017/11/23/elections-locales-sans-enthousiasme-enalgerie 5219039 3212.html [consulta : 14 de junio de 2020].
} 
decisiones políticas. Esto provocaría la paradoja por la cual las mujeres obtendrían cada vez más derechos en política (inclusión), pero permanecerían ausentes de los puestos de decisión (exclusión) (Dris-Aït Hamadouche, 2016: 1).

Además, la falta de celo por parte de las instituciones competentes para controlar las listas electorales, unida a la inexistencia de sanciones comprometen seriamente la efectividad de las cuotas. Este fue uno de los motivos que favorecieron la bajada de la tasa de representación femenina en 2017, ya que algunos partidos ni siquiera respetaron las cuotas mínimas fijadas, tal y como denunciaron algunas organizaciones femeninas (CIDDEF, 2017: 69).

Con el objetivo de influir en la sociedad civil acerca de la necesidad de implicar a las mujeres en la política se han puesto en marcha algunas iniciativas entre las que destaca el Programa de la ONU para el Desarrollo (PNUD) que lanzó en 2013 un plan de apoyo a la participación política efectiva y durable de las mujeres. Con estos objetivos llevaron a cabo una serie de programas educativos en colaboración con el Ministerio del Interior y las colectividades locales para mejorar los conocimientos de las mujeres electas sobre democracia participativa y sobre la gestión de los servicios públicos ${ }^{35}$. Así mismo, el PNUD propuso en 2015 la creación del Foro de Parlamentarias Argelinas ${ }^{36}$, con el objetivo de que mujeres de distintos países pudieran dialogar y desarrollar estrategias comunes para resolver cuestiones que les afectan a todas, como son la defensa de sus derechos y la consecución real de la igualdad.

Finalmente, como balance de la aplicación práctica de la Ley de cuotas, se puede observar que, pese a unos primeros resultados electorales alentadores, el descenso de mujeres electas en los posteriores comicios pone de manifiesto que la Ley de cuotas no está alcanzando el éxito esperado, no sólo por sus propias carencias, sino porque la sociedad no es todavía una sociedad igualitaria y persisten estereotipos de género que consideran la política como espacio masculino mientras que el cuidado de la familia y el hogar siguen recayendo sobre las mujeres que se enfrentan a obstáculos que dificultan la conciliación laboral y familiar.

\section{Conclusiones}

La adopción del mecanismo de cuotas ha provocado un aumento significativo del número de mujeres electas a la vez que ha contribuido a mejorar la imagen exterior de país. La presencia de mujeres en las asambleas nacionales y locales "constituye un orgullo para la sociedad argelina" 37 que en los últimos años ha sido testigo de una serie de mejoras legales en otros ámbitos como la nacionalidad o la criminalización de la violencia en contra de las mujeres. El gobierno, con esta medida, se reafirma en su compromiso constitucional de trabajar en la promoción de los derechos políticos de la mujer, y además se ve recompensado indirectamente por el hecho de que la mayoría de las candidatas electas pertenecen a los dos partidos principales en el poder ${ }^{38}$. Sin embargo, esta realidad se presenta "maquillada", puesto que todavía queda bastante camino para alcanzar la paridad. Por ello, organizaciones feministas como Réseau Wassila, consideran que las cuotas no responden a la necesidad acuciante de consagrar los derechos de los ciudadanos entre los que se incluyen los de las mujeres cuyos derechos como "ciudadanas a parte entera" son cuestionados por el Código de la familia (Dris-Aït Hamadouche, 2016: 3) que sigue vigente a pesar del incremento de mujeres parlamentarias.

\footnotetext{
${ }^{35}$ Como experiencia piloto, fueron seleccionadas 5 wilayas en las que unas 300 mujeres fueron beneficiarias de una formación llevada a cabo por expertos internacionales.

${ }^{36}$ https://www.undp.org/content/undp/fr/home/ourwork/ourstories/un-avenir-politique-pour-les-femmes-enalgerie.html (PNUD Programme des Nations Unies pour le développement) [consulta : 30 de octubre de 2020].

${ }^{37}$ Declaraciones oficiales del Ministro del Interior, Bedoui, ante el director del PNUD (Programa de Naciones Unidas para el Desarrollo), Algérie Press Service:

http://www.aps.dz/algerie/71272-bedoui-la-presence-de-la-femme-dans-les-assemblees-elues-constitue-une-fiertepour-notre-societe-algerienne?tmpl=component\&print=1 , publicado el 18/03/2018 [consulta: 26 de octubre de 2020]. ${ }^{38}$ En las elecciones de 2017, de 118 diputadas, 84 pertenecían al FLN (51) y al RND (33).
} 
La Ley Orgánica que fija las modalidades para aumentar las posibilidades de acceso de la mujer a la representación en las asambleas electas, como su propio nombre indica, es únicamente una medida para "aumentar las posibilidades de acceso" de las mujeres a las asambleas electas. El mecanismo elegido, las cuotas de candidatas en las listas electorales de los partidos políticos, no garantiza en absoluto un resultado positivo en cuanto al aumento del número de candidatas efectivamente electas, aunque puede contribuir de manera favorable a dichos resultados. Por lo tanto, esta ley se sustentaría en la equidad, y su objetivo estaría más en la línea de conseguir la igualdad de oportunidades para acceder a la representación política. En este sentido, el diseño de las cuotas que propone resultaría más equitativo si se hubiese optado desde el inicio por la paridad de candidatos masculinos y femeninos, presentados de forma alternativa en las listas electorales. La elección de este sistema "fuerza a los partidos políticos a romper su tradición de reclutar mayoritariamente a candidatos masculinos para sus listas y los insta a comenzar en serio a reclutar mujeres" (Dahlerup, 2007: 84).

El sistema puesto en marcha con esta ley resulta insuficiente y representa solo una solución transitoria que deberá ser derogada cuando se alcance la igualdad. Por ello, si esta medida no va acompañada de reformas a otros niveles, que creen las condiciones favorables para que las mujeres se interesen y se integren en los partidos, las cuotas pueden acabar convirtiéndose en un recurso permanente de limitada eficacia que no contribuya realmente a mejorar la posición de la mujer en la sociedad argelina ni a favorecer su presencia en las instancias de poder y en los ámbitos de toma de decisiones, donde su ausencia supone de facto su exclusión (Dris-Aït Hamadouche, 2016: 1). Ningún esfuerzo dará fruto si no se empieza por cambiar la situación de las mujeres desde abajo, es decir, desde la posición que ocupa la mujer en el seno de su propia familia. No se puede pedir a las mujeres que luchen por sus derechos políticos si no se satisfacen primero sus necesidades sociales ni se acaba con los estereotipos de género.

\section{Referencias}

ADDI, Lahouari (2006): "Les partis politiques en Algérie", Revue des mondes musulmans et de la Méditerranée, no 111-112, pp. 139-166. DOI : https://doi.org/10.4000/remmm.2868

BENGHABRIT-REMAOUN, Nouria (2012): “Femmes en politique : une minorité en émergence?”, L'Algérie aujourd'hui : Approches sur l'exercice de la citoyenneté, CRASC Cap. IV, pp. 139-163.

BENZENINE, Belkacem (2013): "Les femmes algériennes au Parlement : la question des quotas à l'épreuve des réformes politiques", Égypte/Monde arabe, no 10, pp. 1-17. DOI : https://doi.org/10.4000/ema.3196

BUSTOS GARCÍA DE CASTRO, Rafael (2019): “El "hirak" popular: la nueva revolución argelina”, Revista Argelina, no 8, pp. 27-35 . DOI: https://doi.org/10.14198/RevArgel2019.8.04

CIDDEF (2016): Annuaire Femmes Algériennes en chiffres 2017, disponible en https://www.ciddefdz.com/pdf/autres-publications/annuaire2016.pdf [consulta: 2 de mayo de 2020].

DRIS-AÏT HAMADOUCHE, Louisa (2009) : “L'abstention en Algérie : un autre mode de contestation politique", L'Année du Maghreb, V, pp. 263-273. DOI : https://doi.org/10.4000/anneemaghreb.588 
DRIS-AÏT HAMADOUCHE, Louisa (2016): “Les femmes dans le système politique algérien: entre inclusion sélective et exclusion ciblée", Insaniyat, no 74, pp. 74-86. DOI: https://doi.org/10.4000/insaniyat.16783

DAHLERUP, Drude (2007): “Electoral gender quotas: between equality of opportunity and equality of result", Representation, no 43, 2, pp. 73-92

DOI: $\underline{10.1080 / 00344890701363227}$

DAHLERUP, Drude (2009): “Acerca de las cuotas", Estocolmo, International Institute For Democracy And Electoral Assistance (IDEA) y Universidad de Estocolmo (2020), disponible en https://www.idea.int/data-tools/data/gender-quotas [consulta: 26 de octubre de 2020].

DUBRUELH, Camille (2011): “Algérie: vote d'une loi accusée de limiter la représentativité féminine", Jeune Afrique, 04/11/2011, disponible en https://www.jeuneafrique.com/178711/politique/alg-rievote-d-une-loi-accus-e-de-limiter-la-repr-sentativit-f-minine/ [consulta: 26 de octubre de 2020].

FOUGÈRES, Louis (1963): "La Constitution Algérienne”, Annuaire de l'Afrique du Nord, Vol. 2, París, Éditions du CNRS, pp. 9-21.

GRABA, Ghania (2013): "Impact du pluralisme juridique sur le statut des femmes et sur leur émergence à la citoyenneté", Insaniyat, no 59, pp. 63-74. DOI: https://doi.org/10.4000/insaniyat.13889

GRIBAA, Boutheina (2009): "État de la situation de la participation de la femme a la vie politique en Algérie, au Maroc et en Tunisie", Projet "Renforcement du leadership féminin et de la participation des femmes à la vie politique et au processus de prise de décision en Algérie, au Maroc et en Tunisie, Túnez, Centre de la Femme Arabe pour la Formation et la Recherche (CAWTAR).

INTERNATIONAL INSTITUTE FOR DEMOCRACY AND ELECTORAL ASSISTANCE (IDEA) (2020): "Base de datos de cuotas", Estocolmo, disponible en https://www.idea.int/data-tools/data/gender-quotas [consulta: 26 de octubre de 2020].

LAMAS, Marta (1996): "La perspectiva de género", Revista de Educación y Cultura de la Sección 47 del SNTE, no 8, pp. 14-20.

PÉREZ BELTRÁN, Carmelo (2017): "Pluralismo bajo control: evolución de la Ley de partidos políticos en Argelia", Estudios de Asia y África, vol. 52, 2(163), pp. 255-284, DOI: https://doi.org/10.24201/eaa.v52i2.2318

PÉREZ BELTRÁN, Carmelo (2016): “La ley argelina de partidos políticos de 2012: estudio introductorio y traducción", Revista de Estudios Internacionales Mediterráneos, no 20, pp. 71-94, DOI: http://dx.doi.org/10.15366/reim2016.20.005

PÉREZ BELTRÁN, (1998): “Evolución del movimiento femenino y feminista en Argelia: las mujeres y la política", Estudios de Asia y África, n. XXXIII, no 1, pp. 75-102.

PÉREZ BELTRÁN, Carmelo (1997): Mujeres argelinas en lucha por las libertades democráticas, Granada, Universidad de Granada.

PÉREZ BELTRÁN, Carmelo (1996): “Entre erradicación y diálogo: mujeres argelinas. Crónica 1995", Miscelánea de Estudios Árabes y Hebraicos, Secc. Árabe-Islam, no 45, pp. 203-232.

REMAOUN, Malika (1999): “Les associations féminines pour les droits des femmes”, Insaniyat, no 8, pp. 129-143, DOI: https://doi.org/10.4000/insaniyat.8331

SAAD-ZOY, Souria et al (2010): Femmes, droit de la famille et système judiciaire en Algérie, au Maroc et en Tunisie, UNESCO Publishing.

SAÏFI, Tokia y PANZERI, Antonio (2012): Elections législatives en Algérie. Rapport de la Délégation d'observation des élections 8-12 mai 2012, Parlamento Europeo. 
STORA, Benjamin (2004 [1994]): Histoire de l'Algérie depuis l'indépendance, 1962-1988, vol. 1, París, Ed. La Découverte.

THIEUX, Laurence (2018): "Sociedad civil y cambio político y social en Argelia: evolución de discursos y estrategias", Revista de Estudios Internacionales Mediterráneos, no 25, pp. 79-92, DOI: https://doi.org/10.15366/reim2018.25.005

URIBE ORTOLA, Ainhoa (2013): “Las cuotas de género y su aplicación en España: los efectos de la Ley de igualdad (LO 3/2007) en las Cortes Generales y los Parlamentos Autonómicos", Revista de Estudios Políticos, no 160, Madrid, pp. 159-197.

WORLD ECONOMIC FORUM (2018): The global gender gap report 2018, disponible en http://www3.weforum.org/docs/WEF GGGR 2018.pdf [consulta: 26 de octubre de 2020]. 